\title{
A NeUrociênCIA E A História das Frações
}

\author{
Manoel de Campos Almeida \\ PUCPR - UFPR: EMERITI
}

(aceito para publicação em maio de 2020)

\begin{abstract}
Resumo
O presente trabalho visa investigar como o cérebro processa as frações, sejam elas simbólicas ou não. Estudos recentes principiam a esclarecer os mecanismos neurológicos subjacentes a esse processo. Eles demonstram que frações não são apenas construtos mentais, resultados de considerá-las como a razão entre dois números inteiros, mas sim são dadas intuitivamente, ou seja, são representações inatas. Investiga-se como esses resultados podem ser traduzidos à luz da História da Matemática, mostrando-se como a neurofisiologia do processamento das frações influiu na evolução histórica desse conceito matemático. Enfatiza-se a importância pedagógica desses resultados.
\end{abstract}

Palavras-chave: Matemática, História, Neurociência. Frações.

\section{[NEUROSCIENCE AND THE HISTORY OF FRACTIONS]}

\begin{abstract}
The present work aims to investigate how the brain processes fractions, whether symbolic or not. Recent studies are beginning to clarify the neurological mechanisms underlying this process. They demonstrate that fractions are not only mental constructs, results of considering them as the ratio between two whole numbers, but are given intuitively, that is, they are innate representations. It is investigated how these results can be translated into the light of the history of mathematics, showing how the neurophysiology of the processing of fractions influenced the historical evolution of this mathematical concept. The pedagogical importance of these results is emphasized. The pedagogical importance of these results is emphasized.
\end{abstract}

Keywords: Mathematics, History, Neuroscience. Fractions. 


\section{Preâmbulo}

Cabe, preliminarmente, um esclarecimento sobre o que os gregos interpretavam por multitude. Boécio, em seu De Institutione Arithmetica (MASI, 1983, p.72), nos esclarece que há duas espécies de essências, que compõem todas as coisas. Uma é contínua, denominada magnitude, que caracteriza corpos contínuos, tais como uma pedra, uma árvore ou uma área ou volume. A outra é caracterizada por ser disjunta, determinada por suas partes, reduzida a uma única reunião coletiva, tal como um rebanho, um coro, uma multidão, uma pilha de coisas, cujo nome próprio é multitude. Diríamos, hoje, que magnitude é um conjunto contínuo e que multitude é um conjunto discreto.

\section{Lei de Weber-Fechner}

A lei de Weber estatui que a diferença perceptível entre o tamanho de dois estímulos depende mais da razão entre as suas duas magnitudes do que das suas diferenças absolutas.

Ernest Heinrich Weber (1795-1878) foi precursor em quantificar o modo de como um ser humano responde a um estímulo físico. A Lei que leva seu nome (Lei de Weber) estabelece que uma diferença noticiável entre dois estímulos é proporcional à magnitude dos estímulos. Gustav Theodor Fechner (1801-1887) aperfeiçoou a Lei de Weber, estabelecendo que a sensação subjetiva é proporcional ao logaritmo da intensidade do estímulo.

Essa relação pode ser descrita pela equação diferencial $d p=k \frac{d S}{S}$, onde $d p$ é o acréscimo diferencial na percepção, $d s$ é o acréscimo diferencial no estímulo e $S$ é o estímulo em um dado instante. A constante de proporcionalidade $k$ deve ser determinada experimentalmente. Integrando essa equação, obtemos por solução $p=k \ln S+C$, onde ln denota o logaritmo neperiano (natural) de $S$ e $C$ é uma constante de integração.

Para encontrarmos o valor de $C$, coloquemos $p=0$, isto é, consideremos no instante inicial a ausência de percepções, obtendo daí $C=-k \ln S_{0}$ valor este que substituído na solução obtida e empregando as propriedades dos logaritmos conduz à $p=k \ln \frac{s}{S_{0}}$, o que mostra que a relação entre estímulo e percepção é logarítmica.

Uma consequência da Lei de Weber-Fechner é que a habilidade para discriminar entre dois valores de estímulo depende mais na sua razão do que dos seus valores absolutos. Essa fração, conhecida como fração de Weber, relaciona $S$, um estímulo em um instante considerado, com $S_{0}$, um estímulo inicial. Portanto, a sensação subjetiva é proporcional à fração $\frac{S}{S_{0}}$, ou melhor, ao logaritmo dessa fração (ALMEIDA, 2017, 2019).

\section{Sistemas de representação de valores numéricos}

Presentemente teoriza-se que os animais podem possuir dois diferentes sistemas não verbais para representa valores numéricos. O primeiro sistema (OFM - Object-File Model) 
representa precisamente números pequenos (até 3 ou 4), sendo cada objeto a ser enumerado em um conjunto representado por um único símbolo. Numerosidades (ou o senso numérico) são explicadas por esse sistema. Como a representação da quantidade é exata, animais empregando esse modelo não seguem a lei de Weber ou mostram efeitos de razão.

No segundo sistema, denominado de sistema de números aproximados (ANSApproximate Number System), animais representam aproximadamente números maiores e também possivelmente números menores. Nesse sistema quantidades são representadas como magnitudes mentais, ou seja, não simbólicas, estando sujeitas a efeitos de razão; portanto, quantidades usando este sistema seguem a lei de Weber (BENSON-AMRAM, 2017; ANOBILE, 2018; ALMEIDA, 2018; et al.)

As frações podem ser representadas simbolicamente ou não-simbolicamente. As frações simbólicas podem ser representadas em duas formas: as frações comuns (da forma a/b, onde a e b são inteiros: $1 / 2,1 / 12, \ldots)$ e as frações decimais $(0,5 ; 0,314 ; \ldots)$. Já as frações não simbólicas representam proporções entre magnitudes (p. ex.: comparação entre dois segmentos; duas áreas, etc.).

\section{Origens históricas das frações}

Cabe relembrar que o uso das frações decimais somente se tornou habitual tardiamente na História da Matemática. Apenas após a publicação da obra "La Disme" (1585), de Simon Stevin (1548-1620) o seu emprego se universalizou. Todavia, as primeiras frações que a história registra foram as sexagesimais, ainda hoje de uso extenso em computações de tempo e de ângulos. O sistema sexagesimal já estava em uso pelos sumerianos, os inventores da escrita, antes de 2100 a.C. Os gregos mantiveram a tradição do emprego de frações sexagesimais, que até hoje é empregada. Já os egípcios preferiam operar com frações unitárias.

Por exemplo, quando hoje nós escrevemos $2 \mathrm{~h} 20 \mathrm{~min} 45 \mathrm{~s}$ isso equivale a $(2+$

$\left.\frac{20}{60^{1}}+\frac{45}{60^{2}}\right)$ horas, em notação sexagesimal, em notação decimal teríamos $\left(2+\frac{1}{3}+\frac{1}{80}\right)$ horas, empregando frações unitárias, resultado que dificilmente seria reconhecido no presente.

Logo, considerações históricas nos permitem levantar uma dúvida sobre como muitos dos estudos neuronais atuais sobre frações são conduzidos. Geralmente eles se limitam às frações decimais, costumeiras hodiernamente. Isso é explicado pelo fato de que os participantes desses estudos só conhecem e estão habituados a operar com frações decimais.

Todavia, historicamente, elas só surgem tardiamente, logo dificilmente influenciariam nos processos cognitivos evolutivos concernentes às frações. Isso permite supor que as mesmas são construtos culturais, mentefatos aos quais algum tipo de processo cognitivo, ainda não devidamente esclarecido, merecedor de pesquisa mais aprofundada, se adequa.

RBHM, Vol. 20, nº 39, pp. 51-62, 2020 
Possivelmente, pode-se conjecturar, nesse processo as frações seriam representações não simbólicas de magnitudes analógicas. Do mesmo modo, como uma informação adicional sobre a sua natureza, pode-se admitir que esse processo independe da base do sistema de numeração empregado para notar as frações, seja ele decimal, sexagesimal ou qualquer outro, o que reforça a hipótese anterior.

\section{Como o cérebro processa as frações}

Até recentemente não tínhamos ideia de como o cérebro processa frações; tanto os processos cognitivos neurofisiológicos envolvidos como o local no cérebro onde são processadas constituíam mistérios arcanos. Recentes estudos, realizados por Simon Jacob e Andreas Nieder (2009), começam a desvendar os véus que os encobrem.

A habilidade de codificar magnitudes, de quaisquer espécies, é um pré-requisito imprescindível para o desenvolvimento da matemática. O surgimento da linguagem, a emergência do pensamento simbólico, a invenção da escrita e sua capacidade para armazenar externamente símbolos, tornaram possível que o ser humano pudesse evoluir de uma aproximada representação de magnitudes absolutas, em seus estágios iniciais, para um conceito de número sofisticado.

A introdução de frações comuns, como a razão de dos números inteiros, foi um passo conceitual fundamental avante, ampliando os limites do campo dos números inteiros para tratar com magnitudes. Embora o conceito de números inteiros seja intuitivo e adequado à contagem e à ordenação, apenas com a introdução das frações o sistema numérico tornou-se mais flexível e ganhou em precisão. Porém, questões essenciais permaneciam, tais como: o cérebro humano processa frações automaticamente ou, alternativamente, acessa numerador e denominador em separado?

Sabia-se que magnitudes absolutas eram codificadas por neurônios individuais, os quais emitem picos (pulsos) de descargas elétricas em resposta a determinados números. Contudo, não era conhecido se como a razão de dois números era codificada, se processando o numerador e o denominador em separado, ou pela extensão da codificação de magnitudes às quantidades relativas.

Os resultados de suas pesquisas mostram que frações não são apenas construtos mentais, resultados de considerá-las como a razão entre dois números inteiros, mas sim são dadas intuitivamente, ou seja, são representações inatas.

Os experimentos por eles realizados mostraram que populações de neurônios no córtex parietal, ao redor do sulco interparietal (IPS) anterior e horizontal, são sintonizadas para determinadas frações, independentemente do formato de sua representação. Isso quer dizer que não importa se as frações são representadas como numerais, ou seja, simbolicamente: 1:6, 3:6, 5/, ..., ou são apresentadas em uma forma verbal (1/6 um sexto: sechstel, em alemão; 1/4 um quarto: viertel, etc.). Isso significa que razões simbólicas são representadas como uma categoria de magnitudes abstratas no cérebro humano. Portanto, o córtex interparietal representa frações comuns de um modo independente de sua notação (KADOSH, 2007, 2009; NIEDER, 2004, 2006, 2019; et. al.). 
Desse modo, constatou-se que as regiões do cérebro com populações neuronais ativadas por mudanças nas frações que lhe são apresentadas, tanto simbolicamente como verbalmente, são o sulco interparietal e o córtex pré-frontal.

Esses estudos demonstram que o cérebro humano não executa uma simples divisão para criar um número real, p. ex. $1 / 4=0,25$, processando então números reais ao invés de frações ou proporções, mas sim constituem uma categoria própria de magnitudes abstratas, específica, inata.

Seria de se esperar que resultados similares fossem obtidos quando fossem apresentados como estímulos razões de magnitudes não simbólicas e não verbais; analógicas, portanto. Dados obtidos com primatas não humanos sugerem que macacos também compartilham o conceito de proporcionalidade (frações) quando treinados a discernir entre as razões de dois comprimentos de linha (NIEDER, 2004, 2006; BONGARD, 2010; CANTLON, 2006).

Estudos eletrofisiológicos subsequentes do córtex pré-frontal mostraram fortes similaridades na codificação de quantidades absolutas e relativas; a atividade neuronal decresce gradualmente quando a distância entre um estímulo pré-determinado e um estímulo apresentado aumenta, devido ao efeito SNARC.

Aparentemente, na cultura ocidental tanto o alfabeto como os números parecem ser representados em uma "linha mental" horizontal orientada da esquerda para a direita (o efeito SNARC: Spatial-Numerical Association of Response Codes). Já os chineses letrados associam números a um eixo vertical, o que mostra que fatores outros, como a direção da escrita adotada, podem influir nessa percepção. É interessante observar como a cultura pode influir nesse efeito cognitivo.

$\mathrm{Na}$ maioria dos adultos, a mera apresentação de um numeral arábico já levanta preconceitos na orientação da sua atenção e na sua resposta motora. Mesmo quando executando uma tarefa simples como decidir se um número é par ou ímpar, ou se é maior ou menor que cinco, números pequenos são automaticamente colocados no lado esquerdo do espaço, enquanto que números maiores são mapeados no lado direito. Isso é uma consequência do efeito SNARC (ALMEIDA, 2018).

Numerosas evidências mostram, portanto, que as mesmas áreas do cérebro que processam numerosidades estão também sintonizadas no processamento de frações.

Essa nova compreensão sobre como o cérebro interpreta frações comuns contribui para esclarecer a importância das frações de Weber no tratamento de numerosidades. Como elas são representações inatas, uma categoria própria de magnitudes abstratas, as frações de Weber se constituem em um elemento chave na compreensão de como o cérebro processa numerosidades e do porque ele percebe números em uma escala logarítmica, conhecida como efeito SNARC, pois a percepção é proporcional a essas frações.

\section{Impactos sobre a pedagogia da matemática}

Esses resultados impactam o modo de como a pedagogia da matemática atua no ensino de frações. Sobre o assunto, Jacob e Nieder (2009) assim concluem: 
"Em contraste com proporções não simbólicas, frações são acreditadas serem não intuitivas e difíceis de compreender, apesar do construto semanticamente análogo. Crianças requerem ensino formal para compreender e usar corretamente frações, e constata-se que mesmo adultos tratam o numerador e o denominador em separado, implicando que o termo [fração] é processado por operações separadas sobre inteiros. (...) Aqui, demonstramos que, em ausência de uma tarefa específica, temos acesso uma representação automática de quantidades relativas. Nossos resultados advogam uma visão de frações menos rígida e formal, desencorajando seu uso primário como meros lóculos para números racionais, [ou] decimais. Adotando um enfoque mais instintivo, natural para frações, a educação matemática poderia ser modificada para nos auxiliar a melhor explorar sua formidável base neuronal" (JACOB \& NIEDER, 2009).

Isso abre novas perspectivas sobre o tema para a educação matemática. Técnicas inovadoras de ensino podem ser desenvolvidas, mais naturais e adequadas à nossa estruturação cerebral. Também confirma que a natureza da matemática pode ser mais bem entendida por meio de estudos sobre como nosso cérebro a processa. Igualmente comprova que uma visão multidisciplinar pode propiciar novos insights sobre os processos cognitivos envolvidos, como propugnamos anteriormente (ALMEIDA, 2011, 2019).

\section{Como povos antigos processavam as frações}

O conhecimento de que os humanos processam frações como uma categoria especial de magnitudes abstratas ajuda a compreender porque os povos primitivos, os quais, embora não representassem frações simbolicamente, exprimindo-as talvez verbalmente, concebiam sua existência e desenvolveram técnicas para manipulá-las. Esse fato permite uma melhor compreensão da evolução histórica do conceito de frações.

Esses estudos demonstram que o cérebro humano não executa uma simples divisão para criar um número real, p. ex. $1 / 4=0,25$, processando então números reais ao invés de frações ou proporções, mas sim constituem uma categoria própria de magnitudes abstratas, específica, inata.

Como cada fração é interpretada pelo cérebro por meio de uma representação abstrata, por exemplo, 1/3, lhe é natural considerá-la como submúltiplo de uma unidade numérica de ordem superior, por exemplo: $1=1 / 3+1 / 3+1 / 3$. Isso mostra como nosso cérebro está estruturado para o uso de raciocínios tais como os empregados nas operações aritméticas de povos da antiguidade, em termos de frações unitárias e/ou de transposições de unidades para unidades de menor ordem.

A transposição de unidades para unidades de menor ordem está registrada nos denominados tabletes proto-literatos sumerianos, os mais antigos registros numéricos escritos que possuímos, pois esse povo é considerado o inventor da escrita em torno de 
3500 a.C. Eles não possuíam um único sistema numérico, mas sim vários, cada qual empregado para contar um determinado tipo de coisa.

Um "diagrama de fatores" de cada um desses sistemas indica uma hierarquia de progressivamente maiores unidades e um número de regras de conversão. Contém informação sobre as formas dos signos, nomes e valores das unidades sucessivas de um dado sistema de números e medidas, começando com as unidades mais altas e com os fatores de conversão, os quais expressam quantas unidades de uma dada espécie estão contidas na próxima unidade mais alta. Joran Friberg foi o arquiteto dessa elegante, condensada e elucidativa forma de expressar um sistema metro-numérico (ALMEIDA, 2011).

Por exemplo, o Sistema S usado para contar a maioria dos objetos discretos, i.e., pessoas, animais, peixes, objetos de madeira e de pedra, produtos do leite, tecidos, etc. possuía o seguinte diagrama de fatores:

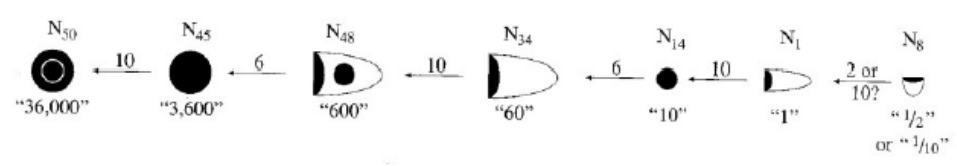

A ideia da transposição de unidades para unidades de menor ordem constitui a arquitetura básica tanto do sistema de trocas de moedas como de qualquer sistema metrológico, e está baseada no fato de que o cérebro interpreta uma fração com uma representação abstrata única.

Os egípcios, cuja escrita foi desenvolvida logo após a sumeriana, estabeleceram um complicado sistema de cálculo onde procuravam expandir qualquer fração em frações unitárias, operando então com elas. Por exemplo, $2 / 21=1 / 14+1 / 42$ ou 2/35 =1/30 + 1/42, expansões do papiro Rhind. Esses são exemplos de como transpor frações para frações unitárias, de menor ordem, neurologicamente mais intuitivas. Contudo algumas de suas expansões eram extremamente complicadas, sendo que até o presente discute-se como eram feitas, o que demonstra a importância que atribuíam a essas frações.

Além disso, contribui para esclarecer os aspectos psicológicos de porque os gregos somente reconheciam como números verdadeiros os números racionais, ou seja, as frações. Tanto os números inteiros como as frações compartilham o fato de que possuem representações abstratas independentes de notações em nosso cérebro. Todavia, comprovou-se que o cérebro humano não executa uma simples divisão para criar um número real, p. ex. $1 / 4=0,25$, processando então números reais ao invés de frações ou proporções. Logo, não deixa de ser inerente à nossa estruturação mental a assunção de que somente os números racionais são verdadeiros. Lembremos a célebre frase de Kronecker: "Deus nos deu os números inteiros, todo o resto é obra do homem".

Isso colabora igualmente para explicar o pavor experimentado pelos gregos quando da descoberta dos irracionais, entidades incomensuráveis, pois estamos 
condicionados, devido à nossa estruturação mental inata, a admitir como naturais somente as operações com números racionais.

Como já mencionado, pesquisas recentes indicam que o processamento de magnitudes não simbólicas (proporcionais) está associado à ativação sulco intraparietal. (IPS). Do mesmo modo, áreas cerebrais associadas ao processamento de números (ou seja, magnitude absoluta) foram ativadas tanto durante o processamento de frações simbólicas, bem como de proporções não simbólicas.

\section{As frações simbólicas e não simbólicas compartilham substratos neurais comuns?}

Estudos conduzidos por Julia Mock e Stefan Huber, em 2018, investigaram o processamento cognitivo de proporções simbólicas (por exemplo, frações e decimais) e não simbólicas (por exemplo, padrões de pontos e gráficos de pizza) em um procedimento de duas etapas. Primeiro, investigaram ativações cerebrais relacionadas ao processamento de proporcionalidades. Em segundo lugar, avaliaram se proporções (frações) simbólicas e não simbólicas compartilham substratos neurais comuns.

Para isso, realizaram um estudo empregando fMRI (Ressonância Magnética Funcional) usando tarefas de comparação de magnitudes com proporções (frações) simbólicas e não simbólicas, utilizando a avaliação do efeito de distância como indicador do processamento de proporções relacionado à magnitudes.

A maior ativação cerebral para o processamento simbólico, quando comparada ao não simbólico, foi encontrada no giro supramarginal direito, no sulco intraparietal e no giro angular esquerdo. Essas regiões são reputadas como importantes durante cálculos exatos e na recuperação de fatos aritméticos, p. ex., durante a adição.

Seus resultados indicaram ativação conjunta de áreas específicas occipito-parietal, incluindo o sulco intraparietal direito (IPS), durante o processamento de proporções de magnitude. Mais especificamente, os resultados indicam que o IPS, que é comumente associado ao processamento de magnitude absoluta (números inteiros), está também envolvido no processamento de informações de magnitude relativa (frações), independentemente do formato de apresentação, seja ele simbólico ou não simbólico, o que confirma parcialmente as suposições levantadas pelos autores anteriormente.

Isso significa que há um substrato neural comum para o processamento tanto de magnitudes relativas, ou seja, de frações, como de números inteiros. No entanto, também encontraram padrões distintos de ativação para o processamento de magnitude de diferentes formatos de apresentação,

Concluíram que seus resultados sugerem que o processamento para formatos de apresentação separados não está associado apenas a manipulações de magnitude no IPS, mas também ao aumento das demandas sobre funções executivas e uso de estratégias, associadas com regiões cerebrais frontais, bem como atenção visual e codificação em regiões occipitais.

Assim, o processamento de proporções (frações) de magnitude pode não refletir exclusivamente o processamento de informações de magnitude numérica, mas também de informações de domínio geral (MOCK, 2018). 
Isso esclarece que esse mecanismo cognitivo poderia também, além de processar proporções (frações) numéricas, igualmente processar proporções não simbólicas de outras grandezas continuas, como área, tamanho, densidade, etc. Lembramos que números são apenas símbolos que expressam quantidades, dessa forma esse mecanismo cognitivo que processa proporções (frações) relativas seria importante parcela estruturante tanto da mente humana como dos animais, confirmando assim a tese de que conceitos matemáticos constituem tijolos fundamentais para construção da mente animal.

Números constituiriam uma estratégia arquitetada pelo homem para avaliar proporções relativas de quantidades, sejam elas discretas ou contínuas. Isso confirma a tese de D’Ambrosio, quando afirmou que a Matemática é uma das estratégias elaborada pelo homem em busca da sobrevivência de sua espécie (D’AMBROSIO \& ALMEIDA, 2017; ALMEIDA, 2017).

\section{Números irracionais}

Resta esclarecer como o cérebro operaria os números irracionais. Como esses números preenchem o contínuo dos reais, poderiam, de alguma forma, serem também tratados por um mecanismo neural geral, que também envolveria proporções (números racionais) de magnitudes. Nesse caso, esse mecanismo teria condições de processar qualquer número real. Contudo, nada é conhecido até o momento sobre isso, em parte devido às dificuldades de se arquitetarem testes que os envolvam.

Não resistimos à tentação de propor aqui um problema. Nosso cérebro está adaptado evolucionariamente a operar com números racionais, ou seja, razões entre números inteiros. Porém, extremamente importante é a razão contendo números irracionais conhecida como razão áurea ou número de ouro.

$$
\phi=\frac{1+\sqrt{5}}{2} \approx 1.618033989 .
$$

Essa razão, embora envolvendo irracionais, parece ser de certo modo intrínseca às nossas noções estéticas de beleza e harmonia. Qual o motivo disso? Porque, além de seu quase onipresente surgimento na natureza, influi em nossas noções estéticas em atividades tão díspares como a arte, a música, o cinema, a arquitetura, etc.? Que circuitos neuronais desperta? Porque nossa estruturação cerebral é por ela ativada? São questionamentos intrigantes, porém certamente merecedores de pesquisas futuras.

\section{A importância do estudo da Pré e da História da Matemática para a compreensão da evolução dos conceitos matemáticos}

Peter Damerow (1939-2011) enfatizou a importância dos estudos históricos para o esclarecimento da evolução dos conceitos matemáticos. Passemos a palavra a Damerow: 
"Eu sou, assim, de opinião que uma consequência necessária do estudo dos processos de aprendizagem da matemática, prevalescentes na teoria da educação matemática, é que os conceitos da psicologia não são suficientes para uma clarificação teórica, porque são incapazes de capturar a dimensão histórica das formas de pensamento matemático" (DAMEROW, Abstraction and Representation, 1998, p. 150 e ss.).

Afirma, com razão, que apenas a psicologia individualmente é impotente para compreendermos a evolução do pensamento matemático, pois não leva em conta o seu desenvolvimento histórico. Continua:

"Na minha visão análise históricas das condições do desenvolvimento do pensamento matemático são necessárias. (...) Para ganhar uma compreensão adequada dos processos de aprendizagem matemática será necessário estudar o desenvolvimento das estruturas de pensamento matemático em termos das mais simples condições de sua manifestação inicial nos processos históricos" (Op. cit., pp. 152-153).

Com isso Damerow queria dizer que devemos voltar nossa atenção para o estudo das primeiras formas de ocorrência das estruturas de pensamento matemáticas no processo histórico, pois surgem em suas formas mais simples, as quais, ao longo do tempo, vão evoluindo, se tornando complexas. Por isso o estudo das primeiras formas de aparecimento das estruturas de pensamento matemático é tão importante, se quisermos realmente compreender o que é matemática, como surgiu, como se desenvolveu, e se pretendermos entender como poderá evoluir.

Em diversas ocasiões o autor teve oportunidade de discutir (e concordar) com Damerow sobre a importância da análise histórica para a compreensão da evolução dos conceitos matemáticos. Infelizmente, Damerow faleceu antes de conhecer os mais recentes estudos da neurociência sobre esses conceitos, lacuna que tentamos preencher.

\section{Bibliografia}

ALMEIDA, Manoel de Campos. Origens da Matemática. Curitiba: Editora Champagnat, 208 p., 1998.

ALMEIDA, Manoel de Campos. Origens da Matemática - A Pré-História da Matemática. vol. II - O Neolítico e o Alvorecer da História. Prefácio por Ubiratan D’Ambrosio. Curitiba: Progressiva, 306 p., 2011.

ALMEIDA, Manoel de Campos. O Nascimento da Matemática - A neurofisiologia e a préhistória da Matemática. São Paulo: Livraria da Física Editora, 2013.

ALMEIDA, Manoel de Campos. A Matemática Na Idade da Pedra. São Paulo: Editora da Livraria da Física, 640 p., 2017.

ALMEIDA, Manoel de Campos. A Gênese do Número - Os Neandertais Sabiam Contar? Curitiba: Manoel de Campos Almeida, 2019. 
ANOBILE, Giovanni; BURR, David C.; IAIA, Marika; MARINELLI, Chiara V.; ANGELELLI, Paola and TURI, Marco. Independent adaptation mechanisms for numerosity and size perception provide evidence against a common sense of magnitude. In: Nature: Scientific Reports, 8:13571, 2018.

ANSARI, Daniel; GARCIA, Nicolas; HAMON, Kathleen and DHITAL, Bibek. Neural correlates of symbolic number processing in children and adults. In: NeuroReport, vol. 16, n. 16, 7 November 2005.

BENSON-AMRAM, Sarah; GILFILLAN, Geoff and McCOMB, Karen. Numerical assessment in the wild: insights from social carnivores. In: Phil. Trans. R. Soc. B 373: 20160508. Access in: <http://dx.doi.org/10.1098/rstb.2016.0508>, 2017.

BONGARD, Sylvia and NIEDER, Andreas. Basic mathematical rules are encoded by primate prefrontal cortex neurons. In: PNAS; vol. 107; $\mathrm{n}^{\circ} 5 ; 2.277-2.282$, February 2, 2010.

CANTLON, Jessica F. and BRANNON, Elizabeth M. Shared System for Ordering Small and Large Numbers in Monkeys and Humans. In:Psychological Science, 17(5), pp. 401406. 2006.

CHOCHON, F.; COHEN L; MOORTELE, P.F. and DEHAENE, S. Differential Contributions of the Left and Right Inferior Parietal Lobules to Number Processing. In: Journal of Cognitive Neuroscience 11:6, pp. 617-630, 1999.

D'AMBROSIO, Ubiratan and ALMEIDA, Manoel de Campos. Ethnomathematics and the Emergence of mathematics. In: The Nature and Development of Mathematics; London: Routledge, 2017.

DAMEROW, Peter. Abstraction and Representation. Berlin: Kluwer, 1998.

DAMEROW, Peter. Prehistory and Cognitive Development. Invited Lecture at the TwentyFifth Annual Symposium of the Jean Piaget Society. Berkeley, June 3, 1995.

DAMEROW, Peter. The Material Culture of Calculation: A Conceptual Framework for an Historical Epistemology of the Concept of Number. Preprint 117, Berlin: Max Planck Institute of History of Science, 1999.

DAMEROW, Peter; SCHMIDT, Siegbert. Aritmetik im historischen Prozess: Wie "naturlich" sind die "naturlich Zahlen"? Preprint 163. Max Planck Institute for the History of Science. 2001.

DEHAENE, Stanislas. The Number Sense. New York: Oxford University Press, 274 p., 1997.

JACOB, Simon N. and NIEDER, Andreas. Notation-Independent Representation of Fractions in the Human Parietal Cortex. In: The Journal of Neuroscience, 29(14)-46524657, April 8, 2009.

JACOB, Simon N. and NIEDER, Andreas. The ABC of cardinal and ordinal number representations. Trends in Cognitive Sciences, vol. 12 n. 2. 2007.

KADOSH, Roi Cohen and WALSH, Vincent. Numerical Representation in the Parietal Lobes: Abstract or not Abstract? In: Behavioral and Brain Sciences. London: Cambridge Press, 2009.

KADOSH, Roi Cohen; LAMMERTYN, Jan and IZARD, Veronique. Are Numbers Specials? In: Progress in Neurobiology 84, pp. 132-147, 2008. 
KADOSH, Roi Cohen; et alii. Notation-Dependent and - Independent Representations of Numbers in the Parietal Lobes. In: Neuron 53, pp. 307-314, January 18, 2007.

KNORR, Wilbur Richard. The ancient tradition of geometric problems. New York, Dover, 1993.

KNORR, Wilbur Richard. Techniques of Fractions in Ancient Egypt and Greece. In: CHISTIANIDES, Jean. Org. Classics in the History of Greek Mathematics. Dordrecht: Kluwer, 2004.

LESLIE Alan M; GELMAN, Rochel and GALLISTEL, C.R. The generative basis of natural number concepts. In: Trends in Cognitive Sciences. vol. 12, n. 5, 2008.

MOCK, Julia; HÜBER, Stefan; BLOECHLE, Johannes and BAHNMUELLER, Julia. Magnitude processing of symbolic and non-symbolic proportions: an fMRI study. In: Behavioral and Brain Functions. DOI: 10.1186/s12993-018-0141-z, December 2018.

MASI, Michael. Boethian Number Theory. Amsterdam: Rodopi, 1983.

MOSKALEVA, Maria and NIEDER, Andreas. Stable numerosity representations irrespective of magnitude context in macaque prefrontal córtex. In European Journal of Neuroscience, vol. 39, pp. 866-874, 2014.

NIEDER, Andreas and MILLER, Earl K. A parieto-frontal network for visual numerical information in the monkey. In: PNAS, n.19. 7457-7462, May 11, 2004.

NIEDER, Andreas; DIESTER, Ilka and TUDUSCIUC, Oana. Temporal and Spatial Enumeration Processes in the Primate Parietal Cortex. In: SCIENCE; vol. 313; 8 September, 2006.

NIEDER, Andreas. Neural constraints on human number concepts. In: Current opinion in neurobiology, 60:28-36, December 2019.

NIEDER, Andreas and MILLER, Earl K. A parieto-frontal network for visual numerical information in the monkey. In: PNAS, n. 19, 7457-7462, May 11, 2004.

NIEDER, Andreas; DIESTER, Ilka; TUDUSCIUC, Oana. Temporal and Spatial Enumeration Processes in the Primate Parietal Cortex. In: SCIENCE; vol. 313, 8 September, 2006.

NIEDER, Andreas. Neural constraints on human number concepts. In: Current opinion in neurobiology, 60:28-36, December 2019.

\author{
Manoel de Campos Almeida \\ PUCPR - Curitiba - Brasil \\ E-mail: manoel1748@gmail.com
}

